\title{
Manage the Traffic Load for Reducing Power Consumption in Wireless Sensor Network with Mobile Sink
}

\author{
Mohammad Khalaf Rahim Al-juaifari \\ University of Kufa, Iraq
}

\begin{abstract}
Most important issues that scientific research seeks to solve the limited power, since consuming battery energy comes from communication among sensor nodes in wireless sensor network.

Anew method for traffic management with load balancing in wireless sensor network (WSN) has been proposed using backbone update messages. The method of reducing the number of connection and maintain these connections by nearby Virtual Backbone (VB) sensors or identify the new VB for maintaining a multi-connections, attach these nodes with new VB to maintain the node connections, analyzed the quality of proposed solutions that implemented with path of sink nodes.
\end{abstract}

Finally, simulation of managing the traffic for sample network focusing on substantial savings by controlling the power by balancing the routing of available connections.

\section{Keywords}

Wireless sensor Network (WSN), Connection Balanced Routing (CBR), Virtual Backbone (VB), Traffic Management Algorithm.

\section{INTRODUCTION}

Met wireless sensor networks field recently increasing interest as a result of the significant expansion in the operation fields and technical developments, as wireless connectivity has helped to ease the spread of information and continuity beyond the field of wireless Internet capability.

Power generating unit is the re-mobilization of energy stocks and practiced by a lot of times even at the expense of processor specifications used in applications that rely on low frequencies and low pattern of energy in addition to the external of flash memory that require space is relatively larger than the device memory static primary.

There are a lot of problems still processing by researchers in the WSN to enhance with various conditions; the most important one is energy consumption.

A sensor network consists of a large number of sensor nodes with multi connections concentrating on some nodes and communicate with their local peers made delay in packet delivery so that multiple techniques has been made such in [1] to enhance the message delivery rate performance using mobile sink in WSN with various purpose. Power can be considered as major issue due to it should be managed to maximize the battery life of sensor. To avoid power dissipation for mobile sink sensors due to using its path rather than others, fair distributed workload is needed.
All nodes including with backbone node connected via one or two hop distance, packet path can be evaluated from source node to destination, so routing required to be considered in multi-hop wireless network. Such a type of VB construction in MANET [2] is concentrated by the researchers in simulations; VB with high transmission range to one hop location from neighbors with VB that started called reserved VB.

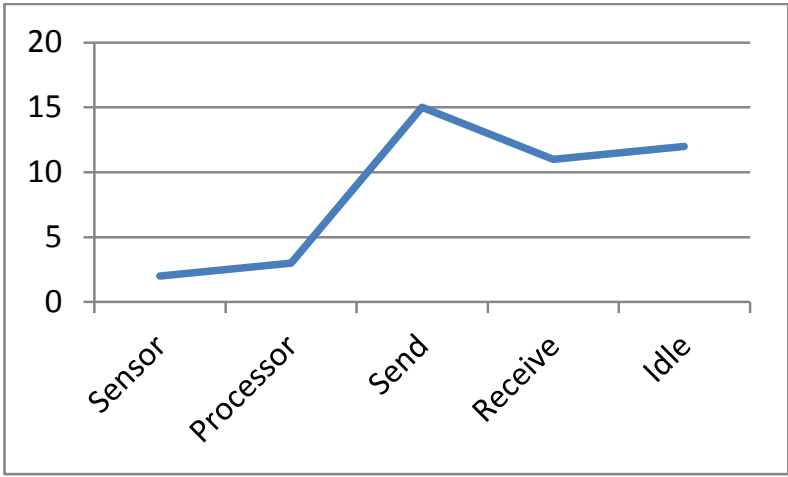

Fig 1: Power Consumption in Micro WATT for WSN Status

Different status of power consumption showed in figure lin which power needed at each specific time to discover a simple but efficient load balancing routing technique, an algorithm to balance the connection without the knowledge of load information and no additional communication overhead. There are several mechanisms for node deployment using Multiple [3] or mobile sink [4] based, multiple sink balance fairly the load among all nodes while one or more number of mobile sink in which power distributed evenly among mobile node.

\section{RELATED WORK}

A practical design of any wireless networks required a balance between an violent reuse of the spectral resource throughout the network and managing the resulting cochannel interference.

In [5] both sleeping and rate adaptation are valuable depending (primarily) on the power profile of network equipment and the utilization of the network itself, while a determining the optimal sense sleep Trees structures in [6] for increasing the monitoring coverage and operational lifetime of mesh-based WSNs.

VBs [7] had been developed and only a few numbers of nodes perform the routing function. By regarding that the number of nodes is attached with unique backbone node during the movement. 
New mechanism for energy management had been developed called context and power aware based task manager [8], The GAF protocol described in [9] a power save protocol that utilizes the information about the geographical location of the nodes. In such protocol like in [10] manage the routes dynamically and balance the data traffic by current load distribution, while in [11] use multi-path routing to fairly divide the traffic among nodes or paths. Load balancing schemes used in many researches to increase network life time and reduce power consumption such as [12],[13],[14],[15],[16],[17], and [18]. Also in [19] data driven routing protocol had been proposed to Optimize network energy by minimize the overhead of link.

In [20] using of distributed congestion control algorithm to balance the transmission rate, proposed system balance the fairly traffic sharing among all sensors path to base station to save power like in [21] with mobile sink. Distributed algorithm in [22] used construct and maintain the connectivity with dominant set in Mobile Ad-hoc Network.

\section{ASSUMPTION AND NETWORK MODEL}

There are different methods to evaluate weights that data traffic from nodes to find the routes. The network sample in this paper is fully connected along with both types of VB and Non-VB nodes which are different in transmission and communication performance and communication protocol, where individual node doesn't address the network load balance. The backbones couldn't manage the routing information for nodes because the nodes including the backbone node connected via one or two hop distance.

Each two nodes, not a single are: single hop connected with direct communication in same messages. The relation between performance and density, Scalability measures the density of the sensor nodes. Collection and processing of data for the sensors is the most efficient in the local data processing as compared with sending raw data to the primary station to deal with them and that's where the largest number of sensors that could lead to flooding in the messages so is choosing some sensors for the purpose of collecting data, which need algorithms assemble require storing messages prior to treatment, and this is a big challenge after the devices with a processing capacity of weak and with limited storage space in the event of large and wide coverage and networks are possible in the event that the amount of data that are sent by each device small degree can be stored sensor as described in figure 2 .

In this paper, An algorithm has been proposed todistribute virtual wireless sensors with three main steps as follow: Construct all VB and maintain its connection, balance the connection of routing, and maintaining the connections, balance energy consumption by routingpackets for each sensor node are needed to neighbors' one hop because of forwarding data by specific nodes will consume more energy than other nodes, VB differs from NonVB in terms of communication and transmission range.

The balance routing algorithm is starting when allow number of connections for every created VB node that connect to the node or reserved one hop for new VB to alternate routing, finally connection maintained and routing table updated by VB. Link weight needed to transmit from one node to another (L) can be calculated by:

$$
L=P \cdot M e / A p
$$

$P_{d}=C \cdot V_{d d}^{2} \cdot f+V_{d d} \cdot I_{o} \cdot \exp \left\{V_{d d} / n^{\prime} V_{T}\right\}$ Where:

Power needed transmit from one node to another "P",

Maximum energy "Me" and available power in node "Ap".

While power consumption in Data Processing (Pd) is calculated as:

Where: "C" represents the total switching capacitance, "Vdd" represents the voltage swing, $\mathrm{F}$ is the switching frequency.

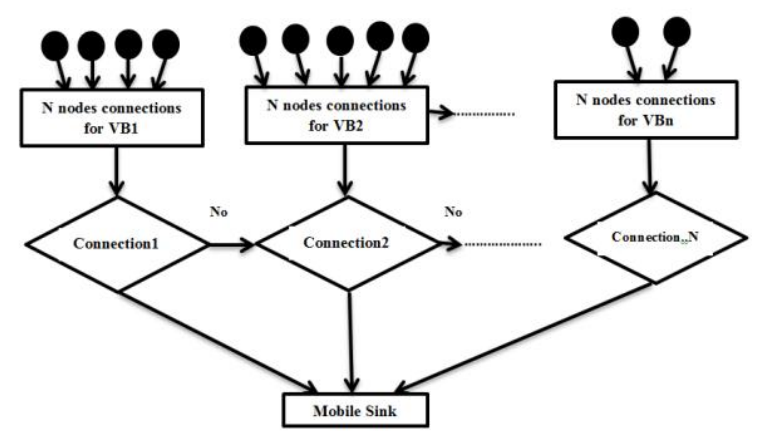

Fig 2: Nodes Connection Mechanism

The following phases are needed to perform the proposed algorithm:

\subsection{Procedure: 1. Connection \& Construction}

2. Input: VB Node VBN, Neighbor Node NN, Non VB node NVBN, Specific Destination D via mobile sink, Transmission Range T, Number of Connections in sample Network C.

\section{3. if (all $N N \& N V B N)$ then}

4. VBN access one hop - location information.

5. end if

6. while (VBN)

7. send packet to required destination.

8. if $(V B N \& D \leq T \& V B N<C)$ then

9. forward packet to $\mathbf{D}$.

10. back to sender to record of $\mathbf{D}$ to routing table.

11. update all information to one hop in routing table.

12. else

13.forward packet to required destination via VBN

"base station" destination.

14. otherwise connection among nodes will be dropped.

15. end if

16. if (VBN) then

17. end if

18. end while

19. if (route path is lost) then

20. create new $\mathbf{C}$ to support backup routing

21. end if 


\subsection{Procedure 2: Connection and routing}

2. Input: VB Node VBN, Network sample N, link among nodes for sample network $\mathbf{L}$, overall node in sample network G, Number of Connections in sample Network $\mathbf{C}$, Reserved VB node RVB, each node in $\mathbf{G}$ can access with one node from these nodes set controller.

3. start first part of routing in $\mathbf{G}$.

4. connect all VBN.

5. while $(V B N \& T)$

6. set controller construction from VBN.

7. if $(\boldsymbol{G}>1)$ then

8. eliminate the node

9. deploy, update, and route a packet using connect set controller of node.

10. stop if there is no node.

11. end if

12. end while

13. repeat all above first part routing steps to cover second part of routing in $\mathbf{G}$.

14. while (node still moving)

15. connections attach to VBN.

16. if $(\boldsymbol{V B N}>C)$ then

17. point towards a new RVB.

18. end if

19. end while

\subsection{Procedure 3: Connection maintenance}

2. Input: VB Node VBN, Number of Connections in sample Network C, Reserved VB node RVB, Neighbor Node NN, overall node in sample network $\mathbf{G}$.

\section{3. while (VBN)}

4. sends $\mathbf{C}$ information to all one hop nodes.

5. originate $\mathbf{V B N}$ by send the attachments of one hop node to other VBN details.

6. if $(\mathbf{C}<=$ Number of cases in $\mathbf{G})$ then

7.VBN used for one RVB for upcoming connection request

8. while (VBN)

9. $\mathbf{C}=$ number of maintenance connections.

10. Share the connections between one RVB with specific case.

11. else ( $\mathbf{C}>$ largest number of connections)

12. load balance need to reevaluate.

13. connect the node to best $\mathbf{N N}$.

14. end if

15. end while

\section{EXPERIMENTAL AND SIMULATION OF RESULT}

The sensor nodes assumed to be distributed randomly, performance measuring for routing protocol and balance the routing via sample network with specifications are: about 100 nodes while simulation above in figure 3 covers about more than 200 nodes , packet=256bytes, specific grid size, and limited power according to transmit/receive packet and start up power required to show blocking rate.

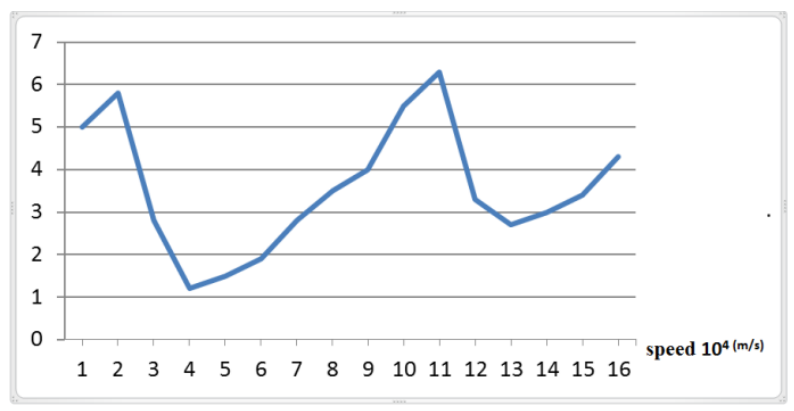

Fig. 3: Graph CBR throughput 106with various speeds

Next, figure 4 demonstrates the balancing of the load among all available paths with greatest bandwidth with respect to speed and packet size. At starting duration of time each node has some time initially to be idle waiting for using entire sample of network in which destination will be selected randomly, packet will be moved to destination node via number of load connections of non VB nodes, figure 4 also shows the relationship between the number of packets those received with regarding to performance of the network and balancing the load starting with speed 10 to the power 4 millisecond's and increasing one by one with time by $\mathrm{x}$-axis and number of delivered packets shows by $y$-axis to describe the performance of proposed system network.

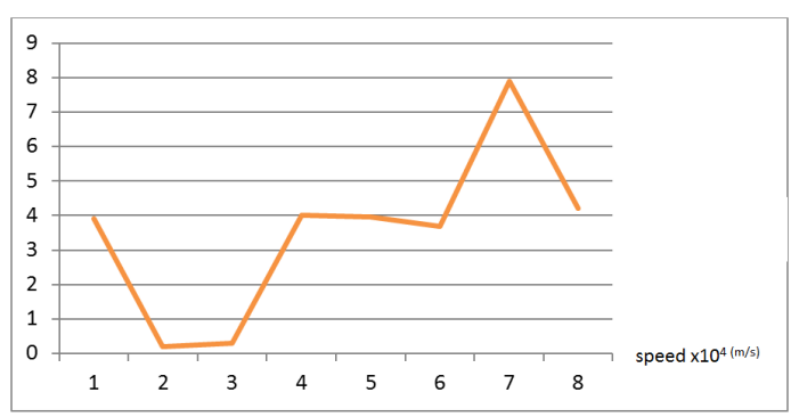

Fig. 4: packet delivery with manage the load performance

\section{CONCLUSION \& FUTURE WORK}

Load balancing enhances energy consumption in mobile node since the movement of these nodes is limited. If the connections are overloaded, a new VB handle this case using network resources with respect to efficient bandwidth blocking rate. VB establishes alternative routing path in case of missing current routing that discover one hop reserved VB.

This paper is designed and implemented based on sample network with limited number of nodes, so that for future work a larger network proposes to build and implement which approximately carry same characteristics of real network. 


\section{REFERENCES}

[1] T. T. Truong1,2, K. N. Brown2 ,C. J. Sreenan1 "Using Mobile Sinks in Wireless Sensor Networks to Improve Building Emergency Response" Royal Irish Academy Research Colloquium, 2010.

[2] H.Y.Yang, C.H.Lin and M.J.Tsai,"Distributed Algorithm for Efficient Construction and Maintenance of Connected K-Hop Dominating Sets in Mobile Ad-Hoc Networks,'IEEETrans.On Mobile Computing, Vol.7, No.4, pp.444-456, APRIL 2008.

[3] Kim H, Kwon T, Mah P. Multiple sink positioning and routing to maximize the lifetime of sensor networks. IEICE Transactions on Communication 2008; 91(11): 3499-3506.

[4] Marta M, Cardei M. Improved sensor network lifetime with multiple mobile sinks. Pervasive and Mobile Computing 2009;5(5): 542-555.

[5] SergiuNedevschi, Lucian Popa, GianlucaIannaccone, "Reducing Network Energy Consumption via Sleeping and Rate-Adaptation", NSDI '08: 5th USENIX Symposium on Networked Systems Design and Implementation.

[6] Rick W. Ha, Pin-Han, X. Sherman Shen, and Junshan Zhang "Sleep scheduling for wireless sensor networks via network flow model"JournalComputer Communications Volume 29 Issue 13-14, August, 2006, Pages 2469-2481.

[7] Y.Sun, X.Gu and J.Qian" Construction of Virtual Backbone on Growth-Bounded Graph with Variable Transmission Range", SEAS TRANSACTION on COMPUTERS, Issue 1, Volume 7, January 2008.

[8] J.Eliasson, P.Lindgren, J.Delsing, S.J.Thompson, and Y.B.Cheng, "A power management architecture for sensor nodes," in Proceedings of the IEEE Wireless Communications and Networking Conference (WCNC '07), pp. 3010-3015, March 2007.

[9] Y. Xu, J. Heidemann, and D. Estrin, "Geographyinformed energy conservation for ad hoc routing", in Proc. IEEE Ann. Int. Conf. Mob. Comp. Netw., Rome, Italy, 2001.

[10] E. Royer and C. Toh, "A review of current routing protocols for ad-hoc mobile wireless Networks," IEEE Personal Communications, Apr. 1999.

[11] J. Broch, D. Maltz, D. Johnson, Y. Hu, and J. Jetcheva, "A performance comparison of multi- Hop wireless ad hoc network routing protocols," in Proc. of the ACM/IEEE Mobicom, October 1998.
[12] F. Bouabdallah, N. Bouabdallah, and R. Boutaba, "LoadBalanced Routing Scheme for Energy-Efficient Wireless Sensor Networks", IEEE GLOBECOM 2008, New Orleans, LA, USA, December 2008.

[13] K. Daabaj, M. Dixon, T. Koziniec, "Experimental Study of Load Balancing Routing for Improving Lifetime in Sensor Networks," the IEEE 5th Conference of Wireless Communications and Mobile Computing (WiCOM'09), Beijing, China, Sept. 24-26, 2009.

[14] C. Wu, R. Yuan, and H. Zhou, "A novel load balanced and lifetime maximization routing protocol in wireless sensor networks", in Proc. of IEEE Vehicular Technology Conference (VTC'08) Spring, Singapore, 2008, pp. 113-117.

[15] J. Gao and L. Zhang, "Load balanced short path routing in wireless networks," in Proc. of IEEE INFOCOM'04, Hong Kong, China, 2004.

[16] H. Dai and R. Han, "A node-centric load balancing algorithm for wireless sensor networks," in GLOBECOM '03, pp. 548-552.

[17] D. Wajgi and N. V. Thakur, "Load balancing based approach to improve lifetime of wireless sensor network," International Journal of Wireless and Mobile Networks (IJWMN), vol. 4, no. 4, pp. 155-167, 2012.

[18] H.-Y. Kim, "An energy-efficient load balancing scheme to extend lifetime in wireless sensor networks," Cluster Computing, pp. 1-5, 2016.

[19] K. Lin, M. Chen, S. Zeadally, and J. J. Rodrigues, "Balancing energy consumption with mobile agents in wireless sensor networks," Future Generation Computer Systems, vol. 28, no. 2, pp. 446-456, 2012.

[20] Brahma, S., Chatterjee, M., Kwiat, K., \&Varshney, P. K. (2012). Traffic management in wireless sensor networks: Decoupling congestion control and fairness. Computer Communications, $\quad 35(6)$, 670-681. doi:10.1016/j.comcom.2011.09.014.

[21] Ishmanov, F.; Malik, A.S.; Kim, S.W. "Energy consumption balancing (ECB) issues and mechanisms in wireless sensor networks (WSNs)": A comprehensive overview. Eur. Trans. Telecommun. 2011, 22, 151-167.

[22] H.Y.Yang, C.H.Lin and M.J.Tsai,'Distributed Algorithm for Efficient Construction and Maintenance of Connected K-Hop Dominating Sets in Mobile Ad-Hoc Networks,'IEEETrans.On Mobile Computing, Vol.7, No.4, pp.444-456, APRIL 2008. 